Koalition überwiegend zum innenpolitischen Bündnis [wurde], das der Regierung Brandt/ Scheel auf wirtschafts- und sozialpolitischem Gebiet den Weg frei räumte“ (S. 613).

Ein Jahr vor Ende der Legislaturperiode hatten sich die Regierungspartner „auseinandergelebt“. Die Wahl von Justizminister Gustav Heinemann (SPD) mit den Stimmen der FDP zum Staatsoberhaupt ließ neuen Koalitionsüberlegungen breiten Raum. Kohl schildert in seinen Memoiren anschaulich, wie sich Kiesinger am Wahlabend über Stunden hinweg als Wahlsieger wähnte, um dann kurz vor Mitternacht alles zu verlieren. Kiesinger hatte es nicht vermocht, die FDP dazu zu bewegen, eine konservativ-liberale Koalition zu bilden. In seinen „25 Thesen über Kiesinger“ resümiert Gassert: „Kiesinger wurde zum Verlierer eines Koalitionspokers und schied mit Bitterkeit aus einem Amt, von dem er selbstbewusst annahm, dass er es nicht lang genug innegehabt hatte, um seine Vorstellungen umsetzen zu können." (S. 757) Die Probleme, mit denen ein Kanzler im Kabinett einer Großen Koalition zu kämpfen hat, können nicht eindrucksvoller dargelegt werden. Es ist zu hoffen, dass Kanzlerin Angela Merkel besonders diesen Teil der Biographie des dritten Kanzlers aufmerksam zur Kenntnis nimmt.

Udo Kempf

\title{
Essayistische Annäherung an die Große Koalition
}

Dittberner, Jürgen: Große Koalition - kleine Schritte. Politische Kultur in Deutschland, Logos Verlag, Berlin 2006, 255 Seiten, $€ 23$,-.

Seitdem Deutschland zum zweiten Mal von einer Großen Koalition regiert wird, ist auch in der Politikwissenschaft das Interesse an dieser Regierungskonstellation wieder gestiegen. Aber was lässt sich in einem Buch, das bereits kurz nach der Entstehung der zweiten Großen Koalition erschienen ist, überhaupt Substantielles über sie sagen? Für eine Bewertung der Politik des jetzigen Regierungsbündnisses ist es zu früh; dieses wird man erst am Ende der Wahlperiode angemessen beurteilen können. Also sollten grundlegende Fragen im Zentrum einer Annäherung an das Thema stehen: Was sind die politischen Voraussetzungen für das Zustandekommen Großer Koalitionen, welche Reformpolitiken sind von ihnen zu erwarten, welcher inneren Dynamik unterliegen sie, wie wirken sie sich auf die Orientierungen und Einstellungen der Bürger aus?

Wer sich eine Antwort auf diese Fragen erhofft, wird von dem Buch von Jürgen Dittberner enttäuscht sein. Statt einer systematischen wissenschaftlichen Analyse erwartet den Leser eher eine essayistische Sammlung persönlicher Eindrücke von politischen Entwicklungen in Deutschland. Dies muss kein Nachteil sein, denn auch gelungene Essays können gute Einblicke in die Problemlagen politischer Phänomene vermitteln, aber diesem Anspruch wird das Buch nur an einigen wenigen Stellen gerecht. Es wirkt über weite Strecken zusammengestückelt, enthält viele Redundanzen und ist außerordentlich fragmentarisch und heterogen.

Der Band beginnt mit einem persönlichen Tagebuch der letzten Wochen der rot-grünen Bundesregierung und streift dann in mehreren Kapiteln verschiedene Perioden der deutschen Politik von den Anfängen der Nachkriegszeit bis zur Gegenwart. Dabei fehlt eine innere Struktur, ein Leitfaden, der die Ausführungen in Richtung des Titels „Große Koali- 
tion“ zusammenführt. Stattdessen lösen sich stark subjektiv gefärbte (keineswegs immer „falsche“) Einschätzungen zur political correctness mit Passagen ab, in denen es das eine Mal um die Niederungen der Kommunalpolitik, das andere Mal um die transatlantische Außenpolitik geht. Dabei lassen sich einzelne Kapitel leicht und flüssig lesen; insbesondere das Kapitel über „Quereinsteiger“ in der Politik, für das exemplarisch die Werdegänge von Angela Merkel und Matthias Platzeck genauer beschrieben werden, enthält eine Reihe gelungener und interessanter Ausführungen.

Es bleibt aber unklar, was Dittberner eigentlich unter „Politischer Kultur“ versteht - immerhin verspricht das Buch im Untertitel, den Begriff im Zusammenhang mit der Regierungskonstellation Große Koalition zu erörtern. So lautet gleich zu Beginn eine These, dass Rot-Grün die „politische Kultur in Deutschland ... neu justiert“ habe (S. 8). Als Beleg werden Tendenzen zur political correctness, zur Personalisierung und Mediatisierung der Politik und zur Zerrüttung des transatlantischen Bündnisses angeführt. Einem anspruchsvollen Begriff von Politischer Kultur, wie er in politikwissenschaftlichen Konzepten (vgl. etwa die Beiträge von Karl Rohe und anderen) entwickelt worden ist, werden diese Beobachtungen nicht gerecht. Wie sich grundlegende politische Orientierungen, Einstellungen und Verhaltensweisen in sozialen Gruppen und Bevölkerungskreisen im Vorfeld der Bildung der Großen Koalition verändert haben und welche Rückwirkung sie möglicherweise auf die Politik der Großen Koalition haben werden - Antworten auf solche Fragen sucht man vergebens. Dabei hat sich gerade an dem unerwartet schlechten Wahlergebnis für die Unionsparteien bei der letzten Bundestagswahl und am Wahlerfolg der Linkspartei gezeigt, dass Sozialstaatsorientierungen jenseits des Diskurses eines großen Teils der politischen und medialen Eliten offensichtlich nach wie vor eine breite Zustimmung in der Bevölkerung genießen - ein Umstand, der sich auch in den bisher sichtbar gewordenen Akzenten der Politik der Großen Koalition niedergeschlagen hat.

Erst im letzten Kapitel des Buches („Perspektiven“) liefert der Autor zum ersten Mal eine etwas präzisere Definition von Politischer Kultur, wenn er schreibt: „,Politische Kultur' ist ein Sammelbegriff für die politischen Institutionen und die allgemein oder partiell gültigen Werthaltungen in einem Gemeinwesen, sofern diese für die Politik relevant sind." (S. 217) In diesem Zusammenhang verweist Dittberner auf die klassischen Studien zur Erforschung politischer Einstellungen in der Bevölkerung von Gabriel Almond und Sidney Verba. Seine folgenden Ausführungen werden dadurch aber nicht überzeugender: So ist die Behauptung, dass „ohne die durch die DDR-Sozialisation aufgebaute USA-Feindlichkeit vieler Ostdeutscher [...] die rot-grüne Außenpolitik nicht möglich geworden [wäre]“ (S. 224), gleich zweifach zu hinterfragen: zum einen daraufhin, ob es überhaupt eine durchgängige USA-feindliche Außenpolitik seitens der rot-grünen Regierung gegeben hat (man denke an die Beteiligung am Krieg in Afghanistan und an Gerhard Schröders Diktum von der „uneingeschränkten Solidarität" nach dem 11. September); zum anderen daraufhin, ob die Differenzen zwischen der Außen- und Sicherheitspolitik der rot-grünen Bundesregierung und der Regierung von George W. Bush auf den besonderen Einfluss der ostdeutschen Bevölkerung zurückgehen. Zwar ist Skepsis gegenüber der Politik der gegenwärtigen Administration in Washington unter ostdeutschen Bürgern stärker ausgeprägt als im Westen, aber grundsätzlich wird sie von einer Mehrheit der Westdeutschen geteilt.

Bedenkenswert sind im letzten Kapitel die Überlegungen und Anregungen zur Reform der verschiedenen politischen Institutionen in Deutschland (Föderalismusreform, Parlamentsreform, politische Führung, Rolle des Bundespräsidenten und Rolle des Bundesver- 
fassungsgerichts). Inwieweit die Große Koalition überfällige Veränderungen in dieser Hinsicht tatsächlich auf den Weg bringen wird, darauf kann das Buch natürlich noch keine Antwort geben.

Lothar Probst

\section{Politisches Denken in Deutschland nach 1945: kontextualisierte Auslegung von Texten}

Greven, Michael Th.: Politisches Denken in Deutschland nach 1945. Erfahrung und Umgang mit der Kontingenz in der unmittelbaren Nachkriegszeit, Verlag Barbara Budrich, Opladen 1 Farmington Hills 2007, 304 Seiten, € 36,-.

Die erstaunliche Fülle des politischen Denkens in der Zeit von 1945 bis September 1949 ist das Thema der eindruckvollen Darstellung von Michael Th. Greven. Der Akzent liegt nicht auf den Zeitschriften, sondern auf den kaum untersuchten selbständigen Publikationen. In fünf thematischen Kapiteln (Politik der geistigen Umkehr und Erziehung, Das Gemeinwesen neu begründen: Varianten des Föderalismus, Gegen Kapitalismus und Kollektivismus: Dritte Wege, Die Sowjetunion als Vorbild, Freiheit im Planstaat) werden Texte von bekannten Autoren wie etwa Friedrich Meinecke, Karl Jaspers, Eugen Kogon und weniger bekannten wie Felix Schottlaender, Hans Peters und Georg Laforet vorgestellt.

Zwei Perspektiven sind leitend: Zum einen, das Markenzeichen von Grevens Publikationen seit Ende der 1990er Jahre, ist es der kontingenztheoretische Ansatz, mit dem gefragt wird, wie offen die Situation nach der vollständigen Kriegsniederlage wahrgenommen wurde. Dass der kommende Kalte Krieg auf erhebliche Weise präsent war und der Atombombeneinsatz dagegen kaum, überrascht. Seltsam berührt, dass nur beim Arzt Rudolf Degkwitz (S. 37) ausdrücklich von jüdischen und russischen Opfern des Nationalsozialismus die Rede ist. Zum anderen stemmt sich das kontextualisierende Vorgehen gegen Lesarten, die von der Position des Nachgeborenen besserwisserisch argumentieren. Beide Perspektiven sind fruchtbar und führen dazu, dass manch ein Urteil revidiert wird. Mehrfach wird das Problem einer Besatzung mit Demokratisierungsabsicht eruiert, das enorme Schwierigkeiten im Verhältnis von Fremd- und Selbstbestimmung in sich birgt (S. 19, S. 25 zum Beispiel).

Greven schreibt keine Geschichte des politischen Denkens. Nicht die ganze Breite interessiert, sondern eine „Symptomatik“ vermittels derer zentrale Fragen diskutiert werden können. Methodisch dominieren hermeneutische Deutungen der Arbeiten von Wissenschaftlern, Schriftstellern, Publizisten und Gelegenheitsautoren. Die Fülle der Einsichten lässt sich nur punktuell andeuten. Eine echte Entdeckung sind die Ideen von Otto Feger, der sich für eine alemannische Demokratie nach Schweizer Vorbild einsetzte. Lesenswerte Darstellungen gibt es zu Alfred Weber, zu Friedrich Meinecke, und mit Blick auf Karl Jaspers sei hervorgehoben, dass dessen Einfluss unmittelbar nach 1945 eine rückwirkende Konstruktion ist, an der Jürgen Habermas wesentlich beteiligt war. Zu den Entdeckungen des Bandes gehört auch Hans Peters, ein tief im Katholischen verankerter Jurist. Seine Bücher thematisieren kulturelle Fragen geistiger Umkehr, er stritt für Föderalismus und für eine wehrhafte Demokratie, die sich von der Weimarer Variante abgrenzt. Insbesondere betonte er die ethischen Grundlagen der Demokratie, und zwar mit Rekurs auf die trefflich charakterisierte US-amerikanische Bewegung für „Moral Re-Armament“ (S. 132 f.). 